R. Kepler, J. Chem. Phys. 39, 3529 (1963). In a triplet exciton system, $\chi_{S} \propto[\exp (-\Delta / T)] / T$ and $T_{1} \propto \exp (\Delta /$ $T)$ where $\Delta$ is the magnetic energy gap. Hence, $\left(T_{1} T\right)^{-1 / 2}$ is proportional to $\chi_{s}{ }^{1 / 2}$ rather than $\chi_{S}$, and $\chi_{S}{ }^{2} T_{1} T$ is proportional to $\chi_{S}$ which is highly temperature depen- dent.

${ }^{19}$ P. A. Lee, T. M. Rice, and P. W. Anderson, Phys. Rev. Lett. 31, 462 (1973).

${ }^{20}$ S. K. Khanna, A. A. Bright, A. F. Garito, and A. J. Heeger, Phys. Rev. B 10, 2139 (1974).

\title{
Photoemission from Rare-Gas Solids: Electron Energy Distributions from the Valence Bands*
}

\author{
N. Schwentner, F.-J. Himpsel, V. Saile, M. Skibowski, $\dagger$ and W. Steinmann \\ Sektion Physik der Universität München, München, Germany \\ and \\ E. E. Koch \\ Deutsches Elektronen Synchrotron DESY, Hamburg, Germany
}

(Received 16 December 1974)

\begin{abstract}
Photoelectron energy distributions for solid $\mathrm{Ne}, \mathrm{Ar}, \mathrm{Kr}$, and Xe have been measured for $8 \mathrm{eV} \leqslant \hbar \omega \leqslant 30 \mathrm{eV}$ by use of synchrotron radiation. From these we obtained the total width and structure of the valence bands. Our data demonstrate that almost all available band-structure calculations fail to predict quantitatively features other than the spin-orbit splitting. Photoelectron energy distributions for Xe in Ar and Xe in Ne support this viewpoint.
\end{abstract}

The electronic structure of rare-gas solids and solid rare-gas mixtures has attracted much interest because of their key role for a better understanding of weakly bound Van der Waals crystals. The optical spectra ${ }^{1}$ of all rare-gas solids show narrow absorption bands, associated with the low-lying excitations of the Ne 2p, Ar $3 p$, $\mathrm{Kr} 4 p$, and $\mathrm{Xe} 5 p$ valence electrons in the vacuum ultraviolet (vuv). They have been successfully interpreted in terms of exciton series converging at the bottom of the conduction band. ${ }^{2}$ From these studies together with photoyield measurements ${ }^{3,4}$ the gap energies and electron affinities have been derived. However, almost no information has been obtained experimentally on the actual band structure, although several calculations by"various methods have been performed. ${ }^{2,5-17}$

We report in this Letter the first photoelectron energy distribution curves (EDC's) for all raregas solids. By varying the excitation energy continuously between 8 and $30 \mathrm{eV}$, we have studied the structure of the valence bands, the influence of the conduction bands on the EDC's, as well as electron-electron scattering processes. In particular we could clarify the discrepancies between the various band-structure calculations.

In our experiments the DESY synchrotron serves as a broad-band vuv light source. The apparatus consists of a normal-incidence mono- chromator and an ultrahigh-vacuum experimental chamber (base pressure $4 \times 10^{-11}$ Torr) with a Heflow cryostat, a reflectometer, and an electron spectrometer. ${ }^{18,19}$ The photoelectron energy analyzer, combination of electrostatic lenses and a retarding grid, was mounted normal to the sample surface (angle of acceptance $3^{\circ}$ ). Counting rates of 1000 counts/sec with a resolution of $200 \mathrm{meV}$ are typical. Charging of the samples, one of the major problems in photoemission of insulators, was minimized by measuring EDC's from thin films (typically 20-100 $\AA$ ).

In. Fig. 1 the EDC's (counting rate versus initial-state energy) are shown for $\mathrm{Ne}, \mathrm{Ar}, \mathrm{Kr}$, and $\mathrm{Xe}$ for several exciting photon energies well below the onset of electron-electron scattering. In this energy range the influence of the electron transport on the EDC's can be neglected because of the large electron escape length (e.g., > 1000 $\AA$ for Ar). ${ }^{18}$ Zero of the binding energy coincides with the top of the valence bands.

The results shown in Fig. 1 contain a large amount of information relevant to the band structure:

Firstly we note that from the EDC's the position of the vacuum level $E_{V}$ can be obtained unambiguously. These values together with the gap energies $E_{G}$ determined from absorption and reflection measurements ${ }^{1}$ result in values for the 


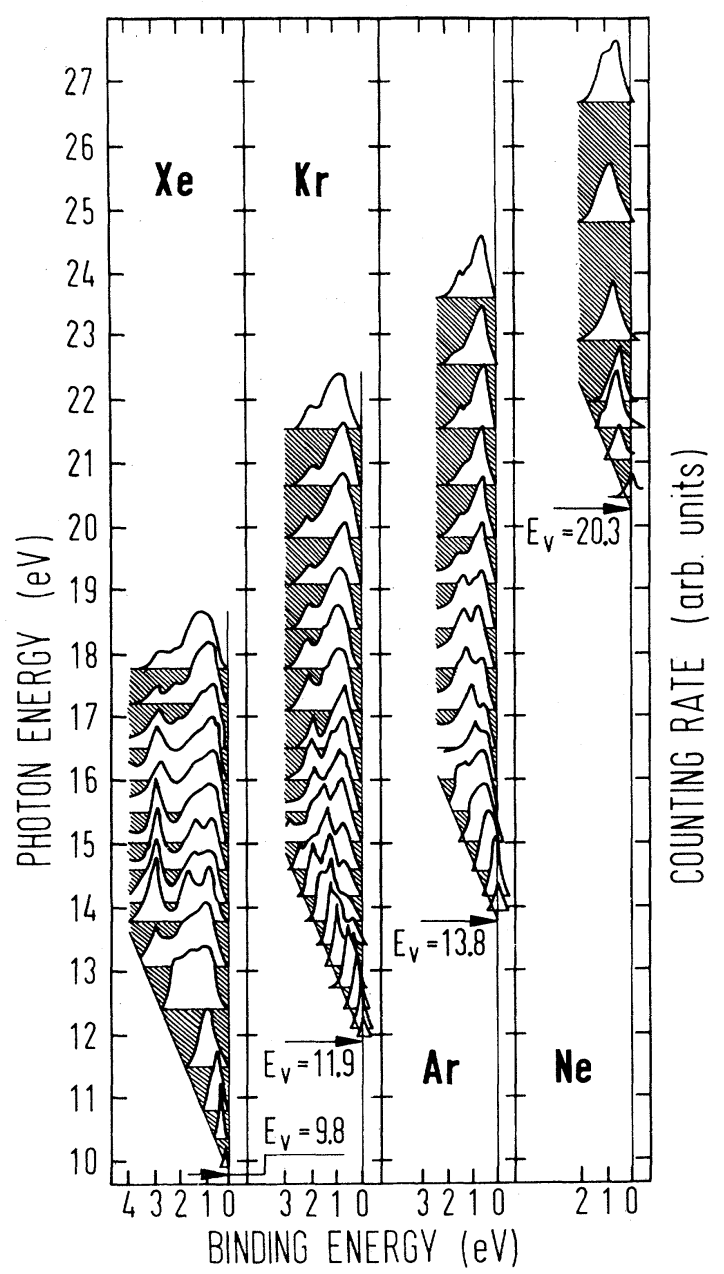

FIG. 1. EDC's for solid Ne, Ar, Kr, and Xe for various photon energies below the onset of electronelectron scattering. Maximum counting rates have been normalized except near threshold. The zerocount line for each individual curve is shifted upwards proportional to the exciting photon energy .

electron affinities $E_{A}=E_{V}-E_{G}$ (see Table I). The results corroborate the values obta ined from earlier photoelectron-yield measurements., ${ }^{3,4,20}$ For $\mathrm{Ne}$ a very large negative value of $E_{A}(\mathrm{Ne})$ $=-1.4 \mathrm{eV}$ is found, confirming the negative value predicted from theoretical estimates ${ }^{21,22}$ and expected from the experimental trend in the $E_{A}$ values for the other rare gases. Previous yield measurements for Ne could give only a lower limit for $\left|E_{A}\right| .^{4}$

Secondly we note that the width of the EDC's, once the photon energy is sufficient to excite electrons from the whole valence band $(23 \mathrm{eV}$ for $\mathrm{Ne}, 17 \mathrm{eV}$ for $\mathrm{Ar}, 15 \mathrm{eV}$ for $\mathrm{Kr}$, and $14 \mathrm{eV}$ for $\mathrm{Xe}$ ), increases systematically from $\mathrm{Ne}$ to $\mathrm{Xe}$
TABLE I. Parameters for the band structure of rare-gas solids as obtained by experiment (expt) and calculation (calc). All energies are in eV. $E_{G}$, bandgap energy; $E_{V}$, vacuum level; $E_{A}$, electron affinity; $E_{\mathrm{s} . \mathrm{o} .}$, spin-orbit splitting; $W_{\mathrm{VB}}$, total width of the valence bands.

\begin{tabular}{|c|c|c|c|c|c|}
\hline & Ref. & $\mathrm{Ne}$ & Ar & $\mathrm{Kr}$ & $\mathrm{Xe}$ \\
\hline$E_{G}{ }^{\text {expt }}$ & a & 21.7 & 14.2 & 11.6 & 9.3 \\
\hline$E_{V}{ }^{\text {expt }}$ & $b$ & 20.3 & 13.8 & 11.9 & 9.8 \\
\hline$E_{A}{ }^{\operatorname{expt}}$ & $\mathrm{b}$ & -1.4 & -0.4 & 0.3 & 0.5 \\
\hline$E_{A}^{\text {calc }}$ & c & -0.6 & 0.3 & 0.62 & 0.39 \\
\hline$E_{\text {s.o. }}{ }^{\operatorname{expt}}$ (gas) & a & 0.14 & 0.22 & 0.67 & 1.31 \\
\hline$E_{\text {s.o. }}{ }^{\text {expt }}$ (solid) & $a$ & $<0.1$ & 0.2 & 0.64 & 1.3 \\
\hline$E_{\text {s.o. }}^{\text {cakc (solid) }}$ & $\mathrm{d}$ & $\cdots$ & 0.2 & 0.7 & 1.37 \\
\hline$W_{\mathrm{VB}} \operatorname{expt}$ & $\mathrm{b}$ & 1.3 & 1.7 & 2.3 & 3.0 \\
\hline \multirow[t]{6}{*}{$W_{\mathrm{VB}}{ }^{\mathrm{calc}}$} & e & 0.4 & 1.0 & 1.6 & 1.8 \\
\hline & $\mathbf{f}$ & 0.4 & 1.3 & 1.6 & $\cdots$ \\
\hline & & $0.65^{\mathrm{g}}$ & $2.3^{h}$ & $1.5^{\mathrm{i}}$ & $1.7^{\mathbf{j}}$ \\
\hline & & $0.5^{k}$ & $1.2^{\mathrm{k}}$ & & \\
\hline & & & $2.6^{1}$ & $2.8^{1}$ & \\
\hline & & & $0.6^{\mathrm{m}, \mathrm{n}}$ & & \\
\hline
\end{tabular}

${ }^{\mathrm{a}}$ Ref. 1 .

b This work.

${ }^{c}$ B. Raz and J. Jortner, Chem. Phys. Lett. $\underline{4}, 155$ (1969).

${ }^{\mathrm{d}}$ See for instance, Ref. 6 .

e Ref. 6 .

fef. 8 .

${ }^{g}$ Ref. 12 .

${ }^{\mathrm{h}}$ Ref. 13.

${ }^{i}$ Ref. 10 .

${ }^{j}$ Ref. 5.

${ }^{\mathrm{k}}$ Ref. 11.

${ }^{1}$ Ref. 9.

$\mathrm{m}_{\text {Ref. } 15 .}$

${ }^{\mathrm{n}}$ Ref. 16.

in accord with the expectations from the increasing atomic spin-orbit splitting (Table I). However, the experimentally determined width, which stays constant with increasing excitation energy, exceeds the value one would expect naively from the spin-orbit splitting of the upper and lower valence bands and the total width obtained for the valence bands in almost all band-structure calculations (see Table I).

Thirdly we note that for Xe a typical EDC curve has three maxima. Because of the smaller spinorbit splitting and smaller total band width, these 


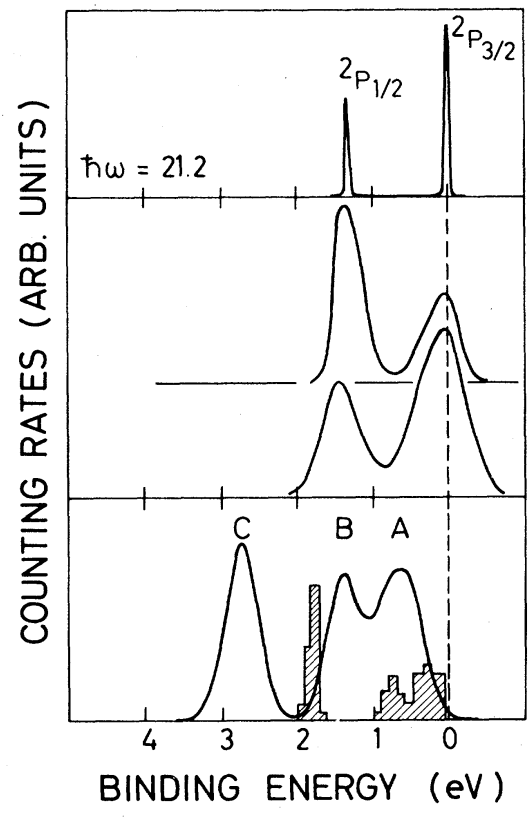

FIG. 2. Bottom: Photoelectron energy distribution curve for solid Xe for $\hbar \omega=13.78 \mathrm{eV}$. The solid curve shows the experimental results; the hatched area shows the result of a calculation by Rössler (Ref. 23). Middle: Photoelectron energy distribution curve for $1 \%$ Xe in $\mathrm{Ar}$ and in $\mathrm{Ne}(\hbar \omega=12.5$ and $17.6 \mathrm{eV}$, respectively). The structureless background due to electrons from the gold substrate has been subtracted. Top: Photoelectron spectrum of gaseous Xe (Ref. 24).

maxima are partly overlapping in $\mathrm{Kr}$, Ar, and Ne. For Xe, EDC's have been calculated by Röss $\operatorname{ler}^{23}$ from the band structure as obtained earlier using the Korringa-Kohn-Rostoker (KKR) method. ${ }^{6,7}$ The energy distribution $N(E, \hbar \omega)$ for direct transitions was obtained by

$$
\begin{gathered}
N(E, \hbar \omega) \propto \sum_{\overrightarrow{\mathrm{k}}} \sum_{C, V} \delta\left[E_{C}(\overrightarrow{\mathrm{k}})-E_{V}(\overrightarrow{\mathrm{k}})-\hbar \omega\right] \\
\times \delta\left[E_{C}(\overrightarrow{\mathrm{k}})-E\right] .
\end{gathered}
$$

Comparison is made in Fig. 2 between the calculated and experimental EDC for $\hbar \omega=13.78-\mathrm{eV}$ incident photon energy. Both experiment and calculation show very similar shapes. In particular, three distinct maxima are obtained. The maxima $A$ and $B$ are associated with excitations from the upper valence bands $\left(j=\frac{3}{2}\right)$ split by the crystal field. The peak $C$ is attibuted to the lower valence band $\left(j=\frac{1}{2}\right)$. The main contributions to these maxima come from flat regions near the Brillouin-zone (BZ) boundary. The experiment gives larger peak separations, $A B$ and $A C$, than does the calculation. This difference is not caused by a different spin-orbit splitting at the center of the $\mathrm{BZ}$ but is rather due to a stronger bending of the bands towards the $\mathrm{BZ}$ boundary than that calculated (see Table I).

In support of this viewpoint and in order to show that the differences are not caused by secondary processes such as electron-phonon scattering we have obtained EDC's from Xe in Ar and $\mathrm{Xe}$ in $\mathrm{Ne}$ (see Fig. 2). They show that the spinorbit splitting of the $n=1$ exciton bands at the $\Gamma$ point of pure Xe and that from Xe in Ar or Xe in Ne environment agrees well with the calculation (about $1.3 \mathrm{eV}$ ). Thus we conclude that for $\mathrm{Xe}$ the width of the valence band is significantly larger $(3 \mathrm{eV})$ than calculated $(1.8 \mathrm{eV}){ }^{6}$ It follows that the effect of $k$ dispersion of the lower $\left(j=\frac{1}{2}\right)$ valence band is about $1.7 \mathrm{eV}$ as determined by our experiment, rather than $0.5 \mathrm{eV}$ as obtained in the calculations. ${ }^{5,6}$

For $\mathrm{Kr}$ the relativistic band-structure calculations $s^{6,10}$ result in a total bandwidth considerably smaller than that observed $(2.3 \mathrm{eV})$. The nonrelativistic bands calculated by Lipari ${ }^{9}$ have a total width of $2.8 \mathrm{eV}$. If one additionally takes into account the spin-orbit splitting, the discrepancy with the observed value would be even worse. The nonrelativistic calculations by Kunz and Mickish, ${ }^{8}$ with a bandwidth of $1.6 \mathrm{eV}$, match the experimental value closely, provided the spinorbit splitting is added. This simple procedure may, however, be too crude. ${ }^{10}$

For Ar the spin-orbit coupling effects are minor. This leads to a fair agreement of the values calculated by Kunz and Mickish ${ }^{8}$ and Dagens and Perrot $^{11}$ with the experiment. The other available calculations are worse (see Table I).

For Ne the measured total width of $1.3 \mathrm{eV}$ exceeds all calculated values considerably. Although in this case the experimental value might be influenced by charging, we can put a lower limit for the total width, that being $1.0 \mathrm{eV}$. This value is still $30 \%$ larger than the value calculated most recently by Euwema et al. ${ }^{12}$ which comes nearest to the experimental result.

Strong changes of the shape and relative intensities occur in the EDC's for different exciting photon energies. They are determined mainly by the conduction bands. Using the parameters for the valence bands as deduced from our experiments one can obtain from these changes estimates for the structure of the density of states in the conduction bands. Thus we would locate the first maximum of the density of states, corresponding to flat regions of the lowest conduc- 
tion band, at $1.1-1.9 \mathrm{eV}$ for $\mathrm{Xe}, 1.6-2.1 \mathrm{eV}$ for $\mathrm{Kr}$, and 2.5-3.5 eV for Ar above the bottom of the conduction band. From modified calculations for the EDC's, considering realistic valence bands, one can extract more detailed information about the structure of the conduction bands, which is difficult to get otherwise. Such calculations are in progress. ${ }^{23}$

At energies equal to the sum of the gap energy and the first exciton energy the onset of electronelectron scattering is observed (not shown in Fig. 1). These energies are $17.8 \mathrm{eV}$ for $\mathrm{Xe}, 21.9$ $\mathrm{eV}$ for $\mathrm{Kr}$, and $26.3 \mathrm{eV}$ for Ar. The cross section for this process has been studied in detail for $\mathrm{Kr}$ and $\mathrm{Ar}$ and will be discussed elsewhere. ${ }^{18,25}$

We would like to thank A. B. Kunz and U. Rössler for helpful discussions and for making available to us unpublished calculated EDC's.

\footnotetext{
*Work supported by Deutsche Forschungsgemeinschaft DFG.

†On leave at Xerox Palo Alto Research Center, Palo Alto, Calif., and University of Illinois, Urbana-Champaign, I11. 61801 .

${ }^{1}$ For a recent review see B. Sonntag and R. Haensel, in "Rare Gas Solids," edited by M. K. Klein and J. A. Venables (Academic, New York, to be published).

${ }^{2}$ For a recent review see U. Rössler, in "Rare Gas Solids," edited by M. K. Klein and J. A. Venables (Academic, New York, to be published).

${ }^{3}$ N. Schwentner, M. Skibowski, and W. Steinmann, Phys. Rev. B $\underline{8}, 2965$ (1973).

${ }^{4}$ E. E. Koch, V. Saile, N. Schwentner, and M. Skibowski, Chem. Phys. Lett. 28, 562 (1974).
}

${ }^{5}$ M. H. Reilly, J. Phys. Chem. Solids 28, 2067 (1967).

${ }^{6}$ U. Rössler, Phys. Status Solidi (b) $\underline{42}, 345$ (1970).

?U. Rössler, Phys. Status Solidi (b) $\underline{45}, 483$ (1971).

${ }^{8}$ A. B. Kunz and D. J. Mickish, Phys. Rev. B $\underline{8}, 779$ (1973).

${ }^{9}$ N. O. Lipari, Phys. Status Solidi $\underline{40}, 691$ (1970), and Phys. Rev. B $\underline{6}, 4071$ (1972).

${ }^{10}$ W. B. Fowler, Phys. Rev. 132, 1591 (1963).

${ }^{11}$ L. Dagens and F. Perrot, Phys. Rev. B $\underline{5}, 641$ (1972).

${ }^{12}$ R. N. Euwema, G. G. Wepfer, G. T. Surratt, and

D. L. Wilhite, Phys. Rev. B 9 , 5249 (1974).

${ }^{13}$ N. O. Lipari and W. B. Fowler, Phys. Rev. B $\underline{2}$, 3354 (1970).

${ }^{14}$ W. B. Fowler, Phys. Rev. B 2, 1591 (1963).

${ }^{15}$ R. S. Knox and F. Bassani, Phys. Rev. 124, 652 (1961).

${ }^{16}$ L. F. Mattheiss, Phys. Rev. A $\underline{133}, 1399$ (1964).

${ }^{17}$ R. Ramirez and L. M. Falicov, Phys. Rev. B 1, 3464 (1970).

${ }^{18}$ N. Schwentner, thesis, Universität München, München, Germany, 1974 (unpublished); N. Schwentner et $a l$, , to be published.

${ }^{19}$ N. Schwentner, A. Harmsen, E. E. Koch, V. Saile, and M. Skibowski, in Vacuum Ultraviolet Radiation Physics, edited by E. E. Koch, R. Haensel, and C. Kunz (Pergamon, New York, 1974) .

${ }^{20}$ Z. Ophir, B. Raz, J. Jortner, V. Saile, N. Schwentner, E. E. Koch, M. Skibowski, and W. Steinmann, J. Chem. Phys. 62, 650 (1975).

${ }^{21}$ L. E. Lyons and M. G. Sceats, Chem. Phys. Lett. $\underline{6}$, 217 (1970).

${ }^{22}$ J. Jortner, Ber. Bunsenges. Phys. Chem. $\underline{75}, 696$ (1971), and references therein.

${ }^{23}$ U. Rössler, private communication.

${ }^{24}$ D. W. Turner, C. Baker, A. D. Baker, and C. R. Brundle, Molecular Photoelectron Spectroscopy (Wiley, New York, 1970).

${ }^{25} \mathrm{~N}$. Schwentner, to be published.

\title{
Self-Consistent Theory of the Chemisorption of $\mathrm{H}, \mathrm{Li}$, and $\mathrm{O}$ on a Metal Surface
}

\author{
N. D. Lang and A. R. Williams \\ IBM Thomas J. Watson Research Center, Yorktown Heights, New York 10598 \\ (Received 20 January 1975)
}

\begin{abstract}
We report the results of a parameter-free, self-consistent, wave-mechanical calculation of the properties of $\mathrm{H}, \mathrm{Li}$, and $\mathrm{O}$ chemisorbed on a metal surface. Comparison is made with available experimental results on dipole moments, adsorption energies, and state densities.
\end{abstract}

We report here the first essentially exact numerical solution of a widely discussed model of chemisorption. The model consists of a semiinfinite uniform distribution of positive charge representing the lattice of substrate metal ions, an atomic nucleus of charge $Z$, and the gas of in- teracting electrons. The equilibrium position of the nucleus and the electron density distribution are evaluated self-consistently by use of the KohnSham local-density description of exchange and correlation. ${ }^{1}$ From this density distribution, we have calculated the dipole moment, heat of ad- 